\title{
Osteosarcoma of maxilla: A case report
}

\author{
Soheyl Sheikh ${ }^{1}$, Shambulingappa Pallagatti ${ }^{1}$, Amit Aggarwal ${ }^{1}$, Deepak Gupta ${ }^{2}$, Nidhi Puri ${ }^{2}$, Amit Mittal $^{3}$ \\ ${ }^{1}$ Prof. Department of Oral Medicine and Radiology, M.M. College of Dental Sciences and Research, Mullana, Ambala, Haryana, \\ India. \\ ${ }^{2}$ Post Graduate student. Department of Oral Medicine and Radiology, M.M. College of Dental Sciences and Research, Mullana, \\ Ambala, Haryana, India. \\ ${ }^{3}$ Associate Professor. Department of Radiodiagnosis and Imaging, M.M. Institute of Medical Sciences and Research, Mullana, \\ Ambala, Haryana, India.
}

Correspondence:

Department of Oral Medicine and Radiology,

M.M. College of Dental Sciences and Research,

Mullana, Ambala, Haryana, India.

E-Mail:drdeepak_26@rediffmail.com

\begin{abstract}
Osteosarcoma is a common primary malignant bone tumor producing osteoid or bone. It occurs very infrequently in the jaws and comprises of $4 \%$ of the tumour number as compared to the osteosarcoma of the long bones. Osteosarcoma of the maxilla is even rarer presenting with common clinical features of pain and swelling. This presented case is a rare case of osteosarcoma of maxilla in a 21-year-old female patient. Diagnosis and pre-operative assessment was performed using a combination of intraoral periapical radiograph, occlusal radiograph, paranasal view and computed tomography (CT). CT revealed destruction of the walls of maxillary sinus and sunray type of ossification in the soft tissues. Conformation was done with histology report followed by surgical removal of the tumor with safety margins. Dental professionals may be the first to detect jaw osteosarcomas in their initial stages. Regardless of the favourable biological behaviour, the patients of jaw osteosarcoma usually exhibit advanced tumor as it often goes unnoticed by the dental professional thus stressing on the need for early diagnosis of the lesion. The aim of this case report is to draw attention to the possibility of diagnosing this tumor based on clinical and radiographical characteristics before confirmation by histology.
\end{abstract}

Key words: Osteosarcoma, tumor, maxilla. 


\section{Introduction}

The term osteosarcoma refers to a heterogenous group of primary malignant neoplasms affecting bone forming or mesenchymal tissues having a histopathological evidence of osteogenic differentiation (1). It is a spindle cell neoplasm and accounts for almost $40-60 \%$ of all bone sarcomas (2). About $60 \%$ of all osteosarcomas occur in the second decade of life in children and adolescents, and about $10 \%$ occur in the third decade. Further, in the fifth and sixth decades, they are frequently secondary to either radiation therapy or transformation in a pre existing benign condition, such as Paget's disease (3).

There is no gender preponderance in osteosarcoma (3) while some authors state that males are affected 1.5 to 2 times as often as females (2). It has a predilection for metaphyses of long bones and the most common sites of involvement are the distal femur, proximal tibia, and proximal humerus (3). This case require special attention because the occurrence of osteosarcoma is quite infrequent in the jaws $(1,2,4)$ and consists of $8 \%$ of the tumor number(1). Jaw lesions usually occur at an older age in fourth decade $(1,4,5)$ while non jaw lesions occur in second decade of life (1). Further mandibular osteosarcomas are more frequent than those in the maxilla (5). A rare few cases arise from within the bone.

It is very important to diagnose osteosarcoma at an early stage due to its continuous infiltrative growth (2). Because of its ability to metastasize via the bloodstream, it is considered as a prototypical mesenchymal malignancy.

This case needs to be reported because these are highly malignant neoplasms with continuous autonomous growth. Although, osteosarcoma possess high metastatic potential and can mutate to more aggressive behaviour, osteosarcoma of the jaw have a relatively low incidence of metastasis and better prognosis (4). Apart of this fact, we come across osteosarcomas in the jaws which have reached higher stages.

In this case report we highlight a rare case of osteosarcoma of maxilla in 21-years-old female patient. The role as well as importance of the oral physician for the early diagnosis and better prognosis of such neoplasms is emphasized.

\section{Case Report}

A 21-year-old female patient presented with a swelling in the upper right back region of the jaw since four months (Fig. 1). It started intraorally and gradually it increased in size and was exhibited extraorally. There was no discharge from the swelling. It was associated with difficulty in opening of the mouth as well as pain on palpation. There was no history of other medical problems and she was otherwise in a state of good health. The rest of the family members were apparently healthy.

She visited a dental practitioner for the same reason two

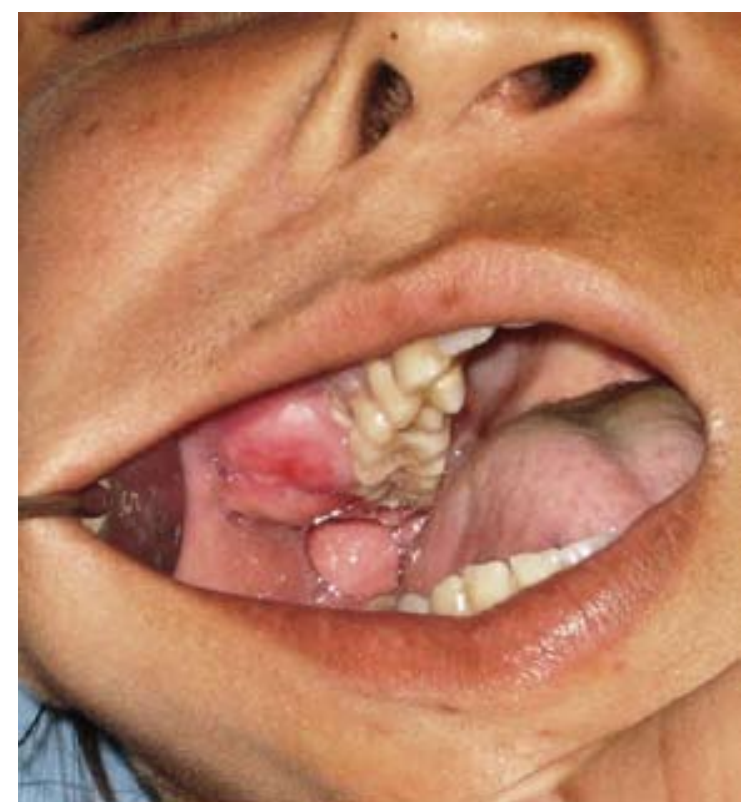

Fig. 1. Intraoral swelling at the right posterior region of the jaw.

months back and was advised for antibiotics and analgesics. There was no permanent relief. In contrast the size of the swelling was increased. The patient was of athletic built and well nourished. All the vital signs like blood pressure, pulse, respiratory rate and temperature were within normal limits.

Extraorally on inspection, a single diffuse swelling was present on the right side of the face over the body of maxilla measuring approximately $3.5 \mathrm{~cm} \mathrm{X} 3 \mathrm{~cm}$ in dimensions. It extended superoinferiorly from the infraorbital margin to $1 \mathrm{~cm}$ above inferior border of mandible and mediolaterally from ala of the nose to $1.5 \mathrm{~cm}$ in front of the tragus of the ear. The skin over the swelling was normal in appearance. The surface over the swelling showed no secondary changes like scar formation, sinus or fistula with no discharge of any kind. On palpation all the inspectory findings were confirmed. There was no localized rise in temperature. The swelling was firm, tender, smooth and immovable and was fixed to the underlying bone. Skin overlying the swelling was slightly stretchable.

On intraoral examination, a solitary localised swelling was seen (Fig. 1) in right buccal vestibule of maxilla measuring $3 \mathrm{~cm} \mathrm{X} 4 \mathrm{~cm}$ in dimensions extending anteroposteriorly from first premolar to third molar and superoinferiorly obliterating the buccal vestibule up to cervical third of the molars. A solitary diffuse swelling was also seen on palate extending anteroposteriorly from second molar to third molar of right side and medially almost to midline in third molar region. The surface of the swelling was lobulated and red in colour with surface ulcerations. On palpation all the inspectory findings were confirmed. The swelling was firm in consistency, non tender and fixed to the underlying bone. It was smooth, non fluc- 
tuant, non compressible and non reducible in nature. No pulsations were felt on palpation. The molars associated with swelling were vital with grade I mobility.

All these features gave a clinical impression of a neoplastic swelling. To confirm the nature of the swelling, various investigations were carried out. The patient was first subjected to radiographic investigations.

Intraoral periapical radiograph of 16,17 , and 18 revealed dense trabecular pattern (ground-glass appearance) of the bone around the apices of the teeth obscuring the tips of the roots with widening of periodontal ligament space and loss of lamina dura (Fig. 2).

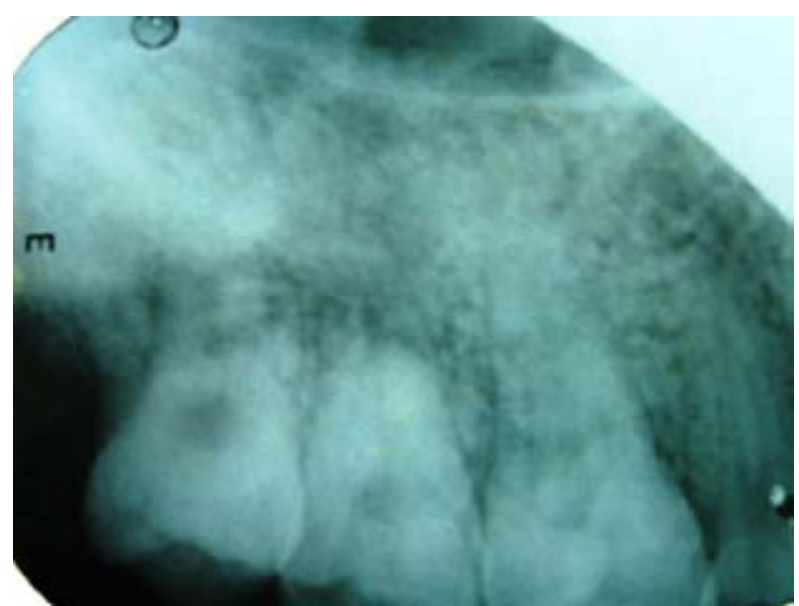

Fig. 2. Periapical radiograph of 16,17, and 18. Dense trabecular pattern of the bone obscuring the tip of the roots with widening of periodontal ligament space and loss of lamina dura.

Occlusal radiograph revealed the expansion of cortical bone in relation to $16,17,18$ and the maxillary tuberosity. Dense trabecular pattern was seen in the hard palate on the right side.

Paranasal sinus view (Water's view) revealed haziness in the right maxillary antrum. There was irregularity with thickening and sclerosis of the inferior and lateral walls of the sinus and ossification in surrounding tissue. The nasal septum and the nasal cavity were normal.

A non contrast computed tomography (CT) of head was taken by Single Slice Spiral CT scanner (Somatom Emotion, Siemens). It revealed irregular destruction of the alveolar margins of the maxilla with the destruction of inferior, lateral and anterior wall of the right maxillary sinus with large surrounding soft tissue. There was evidence of typical sun-ray spiculation because of ossification in the surrounding soft tissue (Fig. 3).

An incisional biopsy was then performed under local anaesthesia.

Histopathological findings: The hematoxiline-eosine (H\&E) stained section revealed proliferation of atypical osteoblasts exhibiting considerable variation in the size and shape. They showed large deeply stained nuclei arranged in disorderly fashion with tumour osteoid and bone formation.

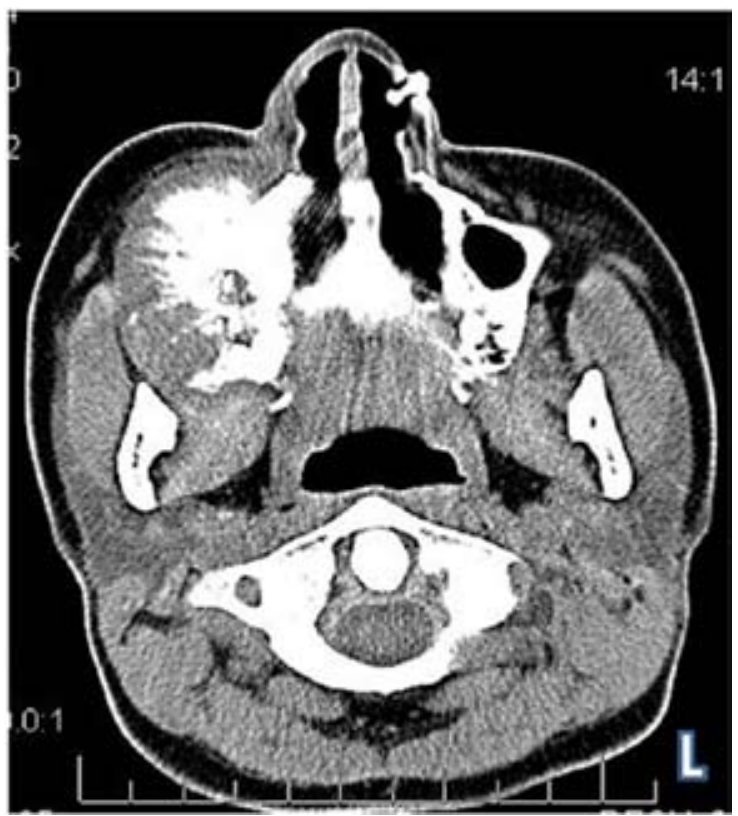

Fig. 3. Axial non contrast CT in soft tissue window revealed destruction of the walls of maxillary sinus with surrounding soft tissue mass and sunray type of ossification in the soft tissues.

Based on the histopathological findings with correlation of radiographic and clinical presentation, the final diagnosis of well differentiated osteogenic sarcoma was made. A chest radiograph was then advised to check for any lung metastasis. The radiograph was completely non significant for metastasis in this case.

Management: The patient underwent complete surgical removal of the tumor with a safety margin of normal bone, followed by chemotherapy as recommended in the literature. The margins in the surgical specimen were not affected by pathology. The surgical wound was then managed by primary closure. Patient is kept on follow up after surgery since last six months during which chest radiograph; CT scan and bone scan were advised to keep a check on recurrence of the lesion. All the reports were normal.

\section{Discussion}

Osteosarcoma is a common primary malignant bone tumour and is a relatively rare disease in the orofacial region $(2,4)$. The diagnosis of osteosarcoma is usually dependent on clinical, radiologic, and histopathologic features (5). The affected patients typically present with pain and swelling of the concerned area (1) along with occasional ulceration (4). Other clinical features include mobility of the tooth, numbness in the lips or other paresthesias (1).

Radiographic evaluation is necessary for diagnosis because clinical symptoms such as pain, paresthesia, swelling etc. are nonspecific $(5,6)$. Radiographically, osteosarcomas may present with an expansion of bone. It may present as a radiopacity, radiolucency or of mixed nature 
(4). There can be widening of the periodontal ligament space (Garrington sign) as seen in our case (1).

There is a need to be more conscious while diagnosing such patients as it often goes undiagnosed for a significant period of time. This is so because some of these signs and symptoms can be produced by a number of different developmental, infectious, benign neoplastic diseases, or malignancies. Further, its presentation is similar to that of osteomyelitis with proliferative periostitis, suppurative osteomyelitis, ossifying fibroma, osteoblastoma, and even fibrous dysplasia, which has often caused an osteosarcoma to be delayed in its diagnosis $(1,4)$.

A plain radiograph reveals a destructive lesion with a moth-eaten appearance, a spiculated periosteal reaction as seen in our case, and a cuff of periosteal new bone formation at the margin of the soft tissue mass known as Codman's triangle (4).

A CT scan of an osteosarcoma often shows formation of irregular endosteal and extracortical bone as well as a destroyed or obliterated cortex. However, CT scan cannot differentiate between osteosarcoma and fibrous dysplasia, a typical sunray spiculations were seen in the soft tissue in this case which were highly suggestive of osteosarcoma (7).

A tissue biopsy is the only means of making a definitive diagnosis and such cases presenting clinically of osteosarcoma requires a biopsy as soon as possible as done in our case. The deep biopsy specimen should be taken from the centre of the lesion $(1,7)$. This will avoid missing the diagnostic portion of the tumor because the superficial specimen will perhaps include the immature part of the lesion (1). The biopsy specimen from the superficial or peripheral aspects of the tumor from the advancing edge is least likely to be representative of the tumour and frequently fail to demonstrate osteoid formation. Further, it will prevent to include benign reactive periosteal bone in the specimen, which could lead to a misdiagnosis.

The histological appearance of osteosarcoma is highly variable. There is the direct formation of osteoid from a sarcomatous stroma as was with our case. The quantity of osteoid and bone that is formed varies considerably, ranging from a sclerotic osseous tumour to one in which multiple sections may be necessary to identify some resemblance of osteoid. The stromal cells may be osteoblastic, chondroblastic, and/or fibroblastic without any prognostic significance.

In general, osteoblastic tumors are most common, but in the jaws the chondroblastic pattern prevails, as was in our case. A myxoid stroma is also frequently seen, and an atypical myxoid proliferation should alert one to the possibility of osteosarcoma.

Early and small lung metastatic deposits are of great concern in osteosarcoma. So its complete screening requires a chest radiograph or a chest CT scan (5). This will either establish the absence of the disease as in our case or early metastasis. In addition, CT scan of the primary site and adjacent structures is suggested for surgical planning $(5,8)$.

It is very obvious that due to common clinical presentation of pain and swelling, the patients with osteosarcoma usually will report to a dental professional first. Despite the adequate skill and knowledge, it is challenging for dental professionals as they usually come across osteosarcoma cases which have reached advanced stages and prognosis in such patients is very poor.

It is concluded that misdiagnosis is very common in osteosarcoma of the jaw. Besides adding to the few cases of osteosarcoma of the maxilla in dental literature, this article would bring attention of the dental professional to approach such cases with greater concern so as to diagnose them at an early stage leading to better prognosis.

\section{References}

1.Yeşilova E, Akgünlü F, Dolanmaz D, Yaşar F, Sener S. Osteosarcoma: a case report. Eur J Dent. 2007;1:60-3.

2.Ong ST, Shim CK, Ng KH, Siar CH. Osteosarcoma presenting as an aggressive nodular mass in the region of the mandible. J Oral Sci. 2004;46:55-9.

3.Tan JZ, Schlicht SM, Powell GJ, Thomas D, Slavin JL, Smith PJ, et al.

Multidisciplinary approach to diagnosis and management of osteosarcoma - a review of the St Vincent's Hospital experience. Int Semin Surg Oncol. 2006;3:38.

4.Amaral MB, Buchholz I, Freire-Maia B, Reher P, de Souza PE, Marigo Hde A, et al. Advanced osteosarcoma of the maxilla: a case report. Med Oral Patol Oral Cir Bucal. 2008;13:E492-5.

5.Bianchi SD, Boccardi A. Radiological aspects of osteosarcoma of the jaws. Dentomaxillofac Radiol. 1999;28:42-7.

6.Forteza G, Colmenero B, López-Barea F. Osteogenic sarcoma of the maxilla and mandible. Oral Surg Oral Med Oral Pathol. 1986;62:17984.

7.Cabral LA, Werkman C, Brandão AA, Almeida JD. Imprint cytology of osteosarcoma of the jaw: a case report. J Med Case Reports. 2009;3:9327.

8.Cavalcanti MG, Ruprecht A, Yang J. Radiological findings in an unusual osteosarcoma in the maxilla. Dentomaxillofac Radiol. 2000;29:180-84. 